\title{
Caracterização clínica e histopatológica de tumores mamários em cadelas atendidas em Viçosa, MG
}

\section{Clinical and histopathological characterization of breast tumors in female dogs taken care of in Viçosa, MG}

Rafael Oliveira David Alves ${ }^{1}$, Vanessa Ferreira da Silva ${ }^{1}$, Lucas Aquino Rodrigues ${ }^{1}$, Daniele Carolina Rodrigues Xavier ${ }^{1}$, Patrick Leonardo Nogueira da Silva ${ }^{2,3}$ (orcid.org/0000-0003-2399-9526), Danillo Velloso Ferreira Murta ${ }^{4}$

1. Curso de Medicina Veterinária pelas Faculdades Unidas do Norte de Minas (FUNORTE), Montes Claros, MG, Brasil. 2. Discente do curso de Pós-Graduação em Enfermagem do Trabalho da Faculdade de Guanambi (FG), Guanambi, BA, Brasil. 3. Mestrando em Saúde, Sociedade e Ambiente pela Universidade Federal dos Vales do Jequitinhonha e Mucuri (UFVJM), Montes Claros, MG, Brasil. 4. Docente do Departamento de Medicina Veterinária das Faculdades Unidas do Norte de Minas (FUNORTE), Montes Claros, MG, Brasil.

\section{Resumo}

Introdução: a neoplasia da glândula mamária é o tumor mais comum em cadelas. Devido a sua elevada frequência, torna-se necessário investigar os aspectos anátomo-patológicos dessas neoplasias de modo a promover o aumento da longevidade e melhorias na qualidade de vida destes animais. objetivo: analisar as características clínicas e histopatológicas de tumores mamários em cadelas atendidas em Viçosa, MG. Métodos: trata-se de um estudo descritivo, exploratório, observacional, com abordagem quantitativa, realizada em um laboratório de histopatologia e histologia com 12 cadelas que apresentam tumor mamário. As amostras analisadas foram coletadas no Hospital Veterinário da cidade de Viçosa, MG. Após a fixação, o material foi avaliado macroscopicamente e, em seguida, submetido ao processamento histológico. As preparações histológicas foram analisadas por microscopia óptica. Resultados: no estudo, verificaram-se 58,3\% de neoplasias malignas e 33,3\% de neoplasias benignas. A maior parte dos tumores da glândula mamária canina foi de comportamento maligno, e o tipo histológico correspondente mais prevalente foi o carcinoma $(91,6 \%)$. Conclusão: os tipos histológicos malignos de maior ocorrência nas cadelas correspondem ao carcinoma. 0 seu diagnóstico e terapêutica precoces são importantes na prevenção do desenvolvimento neoplásico nestas cadelas.

Palavras-chave: Glândulas mamárias animais. Neoplasias. Histologia. Patologia.

\begin{abstract}
Introduction: mammary gland neoplasm is the most common tumor in bitches. Due to its high frequency, it is necessary to investigate the anatomopathological aspects of these neoplasias in order to promote the increase of longevity and improvements in the quality of life of these animals. Objective: to analyze the clinical and histopathological characteristics of breast tumors in female dogs taken care of in Viçosa, MG. Method: this is a descriptive, exploratory, observational study with a quantitative approach, carried out in a laboratory of histopathology and histology with 12 female dogs presenting breast tumor. The analyzed samples were collected at the Veterinary Hospital from city of Viçosa, MG. After fixation, the material was macroscopically evaluated and then subjected to histological processing. Histological preparations were analyzed by optic microscopy. Results: in the study, 58.3\% of malignant neoplasias were observed, accompanied by $33.3 \%$ of benign neoplasy. Most tumors of the canine mammary gland were malignant and the most common histological prevalent was carcinoma (91.6\%). Conclusion: the most frequent malignant histological types in dogs correspond to carcinoma. Its early diagnosis and therapy is important in preventing neoplasm development in these bitches.
\end{abstract}

Key words: Mammary glands. Animal. Neoplasms. Histology. Pathology

\section{INTRODUÇÃO}

As neoplasiasmamárias caninas representam, aproximadamente, $52 \%$ de todas as neoplasias que afetam as fêmeas, e cerca de $50 \%$ dos tumores mamários apresentam malignidade ${ }^{1}$. O tecido mamário, por sua heterogeneidade celular, tornase uma estrutura complexa, controlada por ação hormonal e por várias condições, como crescimento, fatores nutricionais, epidemiológicos, clínico-patológico, bioquímicos e genéticos ${ }^{2,3}$.

As neoplasias mamárias são diagnosticadas de acordo com idade, histórico reprodutivo e sinais clínicos, como a presença de massa mamária e o aumento de volume de linfonodos regionais ${ }^{4}$. A quantidade de glândulas mamárias e as posições em que estão localizadas são determinadas geneticamente nas espécies. Nos cães, são mais comuns cinco pares de glândulas ${ }^{5}$.

A análise microscópica dos tecidos é um método importante no diagnóstico das enfermidades, proporciona verificação minuciosa que abrange as particularidades celulares até a arquitetura tecidual; com esta finalidade, torna-se imprescindível a utilização da histopatologia ${ }^{6}$.

O exame histopatológico é o método de diagnóstico utilizado para identificar as características de uma neoplasia. Além de facilitar a classificação do tumor, o exame histopatológico

Correspondência: Patrick Leonardo Nogueira da Silva. Av. Governador Nilo Coelho, s/n. Bairro São Sebastião Cidade: Guanambi- BA. CEP: 46430-000. E-mail: patrick_mocesp70@hotmail.com

Conflito de interesse: Não há conflito de interesse por parte de qualquer um dos autores.

Recebido em: 30 Abr 2017; Revisado em: 31 Ago 2017;21 Out 2017; Aceito em: 30 Out 2017 
permite verificar o grau de malignidade, a presença de necrose, invasão linfática e vascular ${ }^{7}$. O prognóstico de animais que apresentam neoplasias mamárias depende de vários fatores, entre os quais, destaca-se o estádio tumoral, tipo de células neoplásicas, comportamento clínico do tumor e condição clínica do animal. A Classificação dos tipos histológicos de tumores mamários e seu perfil clínico revelam informações do prognóstico de neoplasias ${ }^{8}$.

As neoplasias mamárias em cadelas apresentam alta prevalência e alto índice de óbitos por, muitas vezes, não serem diagnosticadas precocemente. A literatura científica encontrase escassa quanto a dados recentes sobre esta temática. Considerando os escassos recursos terapêuticos das neoplasias mamárias da cadela e a deficiência de dados na espécie canina, justifica-se a realização deste estudo a fim de contribuir com a investigação de novos dados clínicos e patológicos de cadelas permitindo o estabelecimento de novas abordagens quanto ao diagnóstico e à terapêutica precoces, bem como a melhoria do prognóstico e da qualidade de vida canina.

Este estudo objetivou analisar as características clínicas e histopatológicas de tumores mamários em cadelas atendidas em Viçosa, MG.

\section{MÉTODOS}

Trata-se de um estudo descritivo, exploratório, observacional, transversal, com abordagem quantitativa, realizada no Laboratório de Histopatologia e Histologia do Departamento de Medicina Veterinária da Universidade Federal de Viçosa (DVT/UFV), na qual foram analisados cortes histológicos de tumores mamários provenientes de amostras colhidas em procedimentos cirúrgicos no Hospital Universitário de Medicina Veterinária da UFV (HUVT/UFV). A amostra do estudo é classificada como não probabilística por conveniência.

Em seguida, foi realizada a análise das fichas de avaliação oncológica de tumores de mama estabelecida pelo HUVT/UFV. Por meio das fichas, foi possível coletar dados a respeito dos sinais clínicos locais, do diâmetro, número de nódulos e da glândula mamária envolvida. Durante os meses de setembro e outubro de 2015, foram atendidas no HUVT/UFV 102 cadelas; entre estas, houve 12 casos de tumores mamários. 0 tratamento estatístico se deu por meio de estatística descritiva.

Durante a pesquisa no HUVT/UFV, foram colhidas, aleatoriamente, amostras de neoplasias mamárias de 12 fêmeas. Após a ressecção cirúrgica, os tumores mamários foram coletados e fixados em solução de formol a 10\%. A área de coleta abrangeu a neoformação, bem como a pele, teto e/ou linfonodos envolvidos. Os fragmentos dos tumores mamários foram seccionados longitudinalmente e fixados durante 18 horas em formol $10 \%$, potencial hidrogeniônico $(\mathrm{pH})$ igual a 7,2 . Após isso, conservado em álcool a 70\%. Os fragmentos foram desidratados em soluções alcoólicas crescentes $(70 \%$, $80 \%, 90 \%$ e $100 \%$ ) e, posteriormente, diafanizados em xilol e incluídos em Paraplast Plus (Sigma) ${ }^{9}$.
Os cortes histológicos foram realizados a $5 \mu \mathrm{m}$ de espessura em micrótomo de rotação LEICA e corados segundo as técnicas de Hematoxilina-Eosina (H\&E). As preparações histológicas foram analisadas sob a microscopia óptica e o diagnóstico realizado quando identificada a neoplasia e sua classificação quanto ao seu comportamento biológico e histogênese.

Os dados das fichas de atendimento foram representados por meio de tabela, elaborada pelo programa Microsoft Excel ${ }^{\circledR}$, versão 2010; figuras de cortes histológicos; e gráficos, cujos dados foram armazenados no Statistical Package for the Social Sciences $\left(\mathrm{SPSS}^{\circledR}\right)$, versão 15.0 , e elaborados pelo programa Microsoft Excel ${ }^{\circledR}$, versão 2010.

O estudo obedeceu aos preceitos éticos da Lei Arouca no $11.794^{10}$, de 8 de outubro de 2008, do Decreto no 6.89911, de 15 de julho de 2009, e do Conselho Nacional de Controle de Controle da Experimentação Animal (CONCEA), na qual regulamenta a pesquisa envolvendo a utilização de animais do Filo Chordata, subfilo Vertebrata, para fins de pesquisa clínica. Os procedimentos experimentais que envolveram a manipulação dos animais utilizados foram realizados seguindo rigorosamente as Normas de Conduta para o Uso de Animais no Ensino, Pesquisa e Extensão do DVT/UFV, estando registrado e aprovado no Comitê de Ética da instituição.

O projeto de pesquisa foi apreciado e aprovado pelo Comitê de Ética no Uso de Animais (CEUA) da NOWAVET, sob o Parecer no 74/2015, de 15 de agosto de 2015.

\section{RESULTADOS E DISCUSSÃO}

A avaliação oncológica das cadelas foi realizada por meio da inspeção da cadeia mamária; identificados, por meio de palpação, a sua localização e os sinais clínicos como consistência, forma e/ou presença de ulcerações e aderência. Por meio do paquímetro, foi mensurado o tamanho dos tumores, o número de nódulos e a probabilidade de serem únicos ou múltiplos (Tabela 1). Observou-se, também, que, em uma mesma cadeia mamária, pode haver tumores benignos e malignos, presentes na Figura 1 da lâmina $C$ e na Figura 2 da lâmina $B$, sendo elas da mesma cadeia mamária (Tabela 1 ).

Com o aumento de diagnóstico de tumores de mama em cadelas, o estudo sobre esta temática vem crescendo. Assim, toda alteração nas glândulas mamárias deve ser avaliada para detecção precoce de possíveis neoplasias ${ }^{12}$, uma vez que a região anatômica mais prevalente para o acometimento por neoplasias são as glândulas mamárias ${ }^{13}$.

Os tumores que surgem nas glândulas mamárias frequentemente estão associados ao mamilo ou ao tecido glandular. Os cães normalmente apresentam duas cadeias mamárias, esquerda e direita, composta cada uma por cinco glândulas: torácica cranial direita (D1) e esquerda (E1); torácica caudal direita (D2) e esquerda (E2); abdominal cranial direita (D3) e esquerda (E3); abdominal caudal direita (D4) e esquerda (E4) e inguinal direita (D5) e esquerda (E5). Todas essas glândulas podem ser foco de 
um ou mais tumores, embora as glândulas D3/E3 e D5/E5 sejam mais propícias à evolução de neoplasias, provavelmente, por terem maior quantidade de tecido glandular ${ }^{14}$.

Os achados, após a análise da tabela 1, estão em consonância com a pesquisa de outros autores predominando o foco de tumores nas glândulas D3/E3 e D5/E514. Tumores múltiplos são frequentes em uma única glândula mamária ou pode envolver diversas glândulas mamárias de forma simultânea com diferentes tipos histológicos ${ }^{15}$. O diagnóstico histopatológico de neoplasias contribuiu no desenvolvimento da oncologia veterinária, principalmente no que se refere ao tumor mamário canino, que é a neoplasia mais frequente em cadelas, depois dos cutâneos ${ }^{16}$.

Figura 1. Tipos histológicos de neoplasias mamárias malignas de cadelas que sofreram ressecção cirúrgica no HUVT/UFV. (A) Ficha 1 - carcinoma tubulopapilar. H\&E. 10X. (B) Ficha 2 - carcinoma complexo. H\&E. 10X. (C) Ficha 3 - carcinoma em tumor misto. H\&E. 10X. (D) Ficha 4 - angiossarcoma. H\&E. 10X.
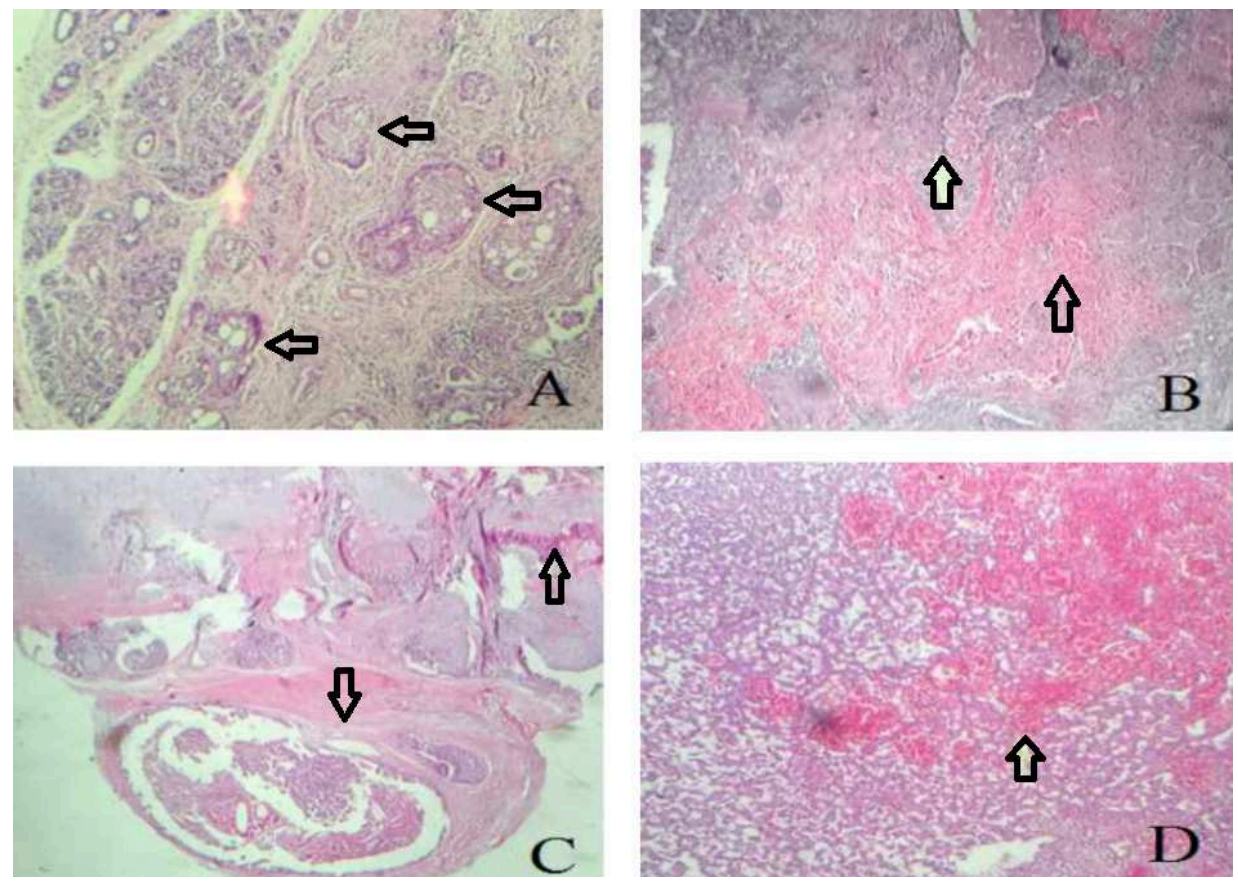

Figura 2. Tipos histológicos de neoplasias mamárias benignas de cadelas que sofreram ressecção cirúrgica no HUVT/UFV. (A) Ficha 5 - condições fibroblásticas. H\&E. 10X. (B) Ficha 3 - linfonodo intramamário. H\&E. 10X. (C) Ficha 6-adenoma complexo. H\&E. 10X. (D) Ficha 7 - lipoma mamário. H\&E. 100X.
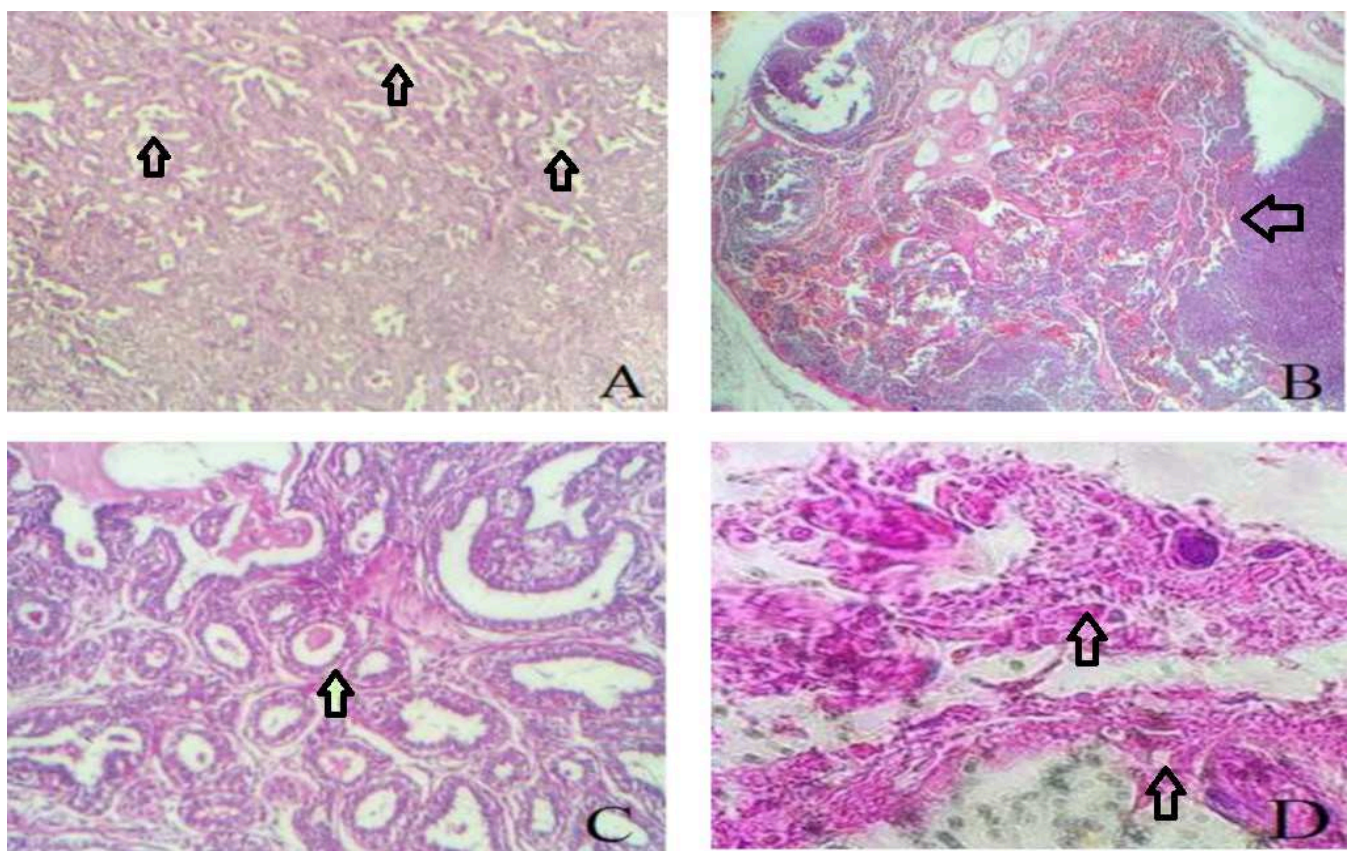

J. Health Biol Sci. 2018; 6(1):17-22 
Tabela 1. Aspectos clínicos e histopatológicos de tumores mamários de cadelas atendidas no HUVT/UFV. Viçosa (MG), 2015.

\begin{tabular}{|c|c|c|c|c|}
\hline \multicolumn{5}{|c|}{ FICHA 1} \\
\hline Glândula mamária & № nódulos/tumores & Diâmetro & Sinais clínicos locais & CPP \\
\hline D2 & Único & $0,5 \mathrm{~cm}$ & Íntegro, firme, regular, não ulcerado e sem galactorreia. & 0,049 \\
\hline D3 & Múltiplos & $1,0 \mathrm{~cm}$ & Íntegros, firmes, regulares, não ulcerados e sem galactorreia. & \\
\hline D4 & Múltiplos & $\begin{array}{r}4,0 \mathrm{~cm} \mathrm{e} \\
1,0 \mathrm{~cm}\end{array}$ & Íntegros, firmes, irregulares, não ulcerados e sem galactorreia. & \\
\hline D5 & Múltiplos & $1,5 \mathrm{~cm}$ & Íntegros, firmes, irregulares, não ulcerados e sem galactorreia. & \\
\hline E5 & Único & $7,0 \mathrm{~cm}$ & $\begin{array}{l}\text { Não aderido, firme, irregular, não ulcerado, metástases distantes } \\
\text { presentes. }\end{array}$ & \\
\hline \multicolumn{5}{|c|}{ FICHA 2} \\
\hline Glândula mamária & № nódulos/tumores & Diâmetro & Sinais clínicos locais & CPP \\
\hline E5 & Único & $5,0 \mathrm{~cm}$ & $\begin{array}{l}\text { Não aderido, firme, irregular, ulcerado com presença de secreção } \\
\text { purulenta. }\end{array}$ & 0,009 \\
\hline \multicolumn{5}{|c|}{ FICHA 3} \\
\hline Glândula mamária & № nódulos/tumores & Diâmetro & Sinais clínicos locais & CPP \\
\hline D1 & Múltiplos & $2 \times 1 \mathrm{~cm}$ & Não ulcerada, macios, regulares. & 0,049 \\
\hline D2 & Único & $2 \times 2 \mathrm{~cm}$ & Não ulcerado, firme e irregular, metástase distante não detectado. & \\
\hline D5 & Único & $0,5 \mathrm{~cm}$ & Não ulcerado, firme e irregular. & \\
\hline E2 & Inúmeros nódulos & 0,5 a $0,2 \mathrm{~cm}$ & Firme, não ulcerado, irregulares. & \\
\hline E5 & Inúmeros nódulos & 0,5 a $0,2 \mathrm{~cm}$ & Firme, não ulcerado, irregulares. & \\
\hline \multicolumn{5}{|c|}{ FICHA 4} \\
\hline Glândula mamária & № nódulos/tumores & Diâmetro & Sinais clínicos locais & CPP \\
\hline D2/E4 & Única & $7,0 \mathrm{~cm}$ & $\begin{array}{l}\text { Íntegra, discretamente irregular, única, sem galactorreia, aderido à } \\
\text { pele. }\end{array}$ & 0,019 \\
\hline E5 & Único & $1,0 \mathrm{~cm}$ & $\begin{array}{l}\text { Firme, regular, não aderido, sem galactorreia, metástase distante } \\
\text { detectada. }\end{array}$ & \\
\hline \multicolumn{5}{|c|}{ FICHA 5} \\
\hline Glândula mamária & № nódulos/tumores & Diâmetro & Sinais clínicos locais & CPP \\
\hline D5 & Único & $2,0 \mathrm{~cm}$ & $\begin{array}{l}\text { Nódulo de consistência firme, não aderido, regular, não ulcerado, } \\
\text { metástase distante não detectado. }\end{array}$ & 0,009 \\
\hline \multicolumn{5}{|c|}{ FICHA 6} \\
\hline Glândula mamária & № nódulos/tumores & Diâmetro & Sinais clínicos locais & CPP \\
\hline E5 & Múltiplos & $5,0 \mathrm{~cm}$ & $\begin{array}{l}\text { Não aderido, pendulado, lobulada, ulcerada com secreção purulenta e } \\
\text { fibrina, metástase distante detectado. }\end{array}$ & 0,009 \\
\hline \multicolumn{5}{|c|}{ FICHA 7} \\
\hline Glândula mamária & № nódulos/tumores & Diâmetro & Sinais clínicos locais & CPP \\
\hline D3 & Único & $1,5 \mathrm{~cm}$ & Nódulo não aderido, regular, não ulcerado, macio. & 0,009 \\
\hline
\end{tabular}

Fonte: Fichas de avaliação oncológica do HUVT/UFV. Viçosa (MG), 2015. CPP = Cálculo de Prevalência Pontual.

Na análise histológica, são definidas as neoplasias mamárias como benignas ou malignas. Ao obter o histórico e realizar o exame físico do cão, percebesse-se que o tumor maligno tem um crescimento rápido, infiltrativo nos tecidos vizinhos e apresenta ulcerado. No entanto, o tumor benigno apresenta características de desenvolvimento lento e expansivo, tendo áreas circulares, não adere aos tecidos próximos e contém células bem distinguidas, próximo do tecido normal ${ }^{17}$. De acordo com os dados obtidos da tabela 1 , normalmente, os tumores malignos apresentam o diâmetro maior do que os benignos.
Considerando todos os animais acometidos com neoplasias do HUVT/UFV, foi verificado que os tumores maiores são mais comuns (Gráfico 1), confirmando estudos anteriores realizados no Brasil ${ }^{18}$. Além disso, de acordo com os resultados obtidos no presente estudo, os tumores que apresentam malignidades são maiores, o que reafirma a correlação entre tamanho do tumor e malignidade, também descrito em outra pesquisa ${ }^{19}$.

Na lâmina (C), glândula mamária torácica caudal direita, foi identificado o carcinoma em tumor misto: com arranjos 
de células epiteliais pleomórficas diferenciadas com pouca formação tubular adjacente à área de cartilagem benigna. Apresenta um padrão histológico complexo de origem epitelial e mesenquimal. Alguns podem virar maligno, desenvolvendo carcinoma em tumor misto; outros sujem em tumor misto benigno ${ }^{15}$.

Gráfico 1. Relação percentual entre o tamanho dos tumores benignos e malignos. Viçosa (MG), 2015.

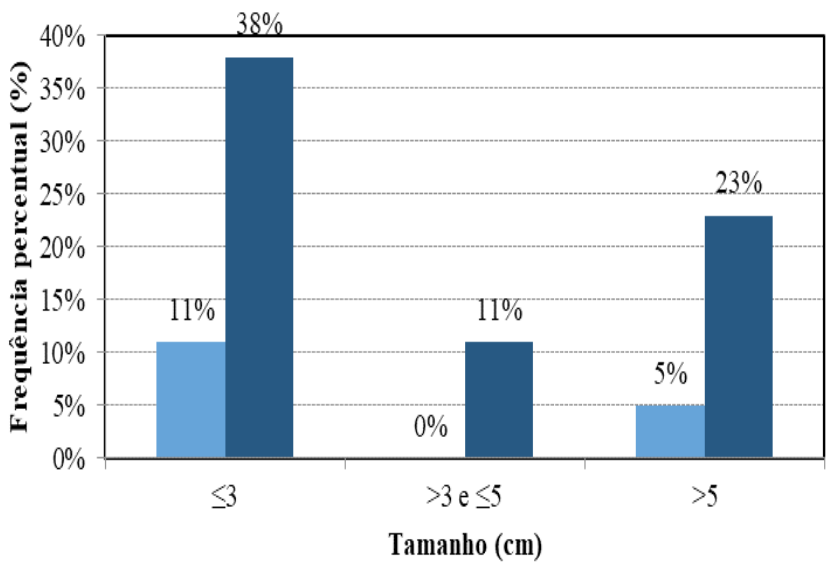

-Benigno $\quad$ Maligno

Na lâmina (B), glândula mamária inguinal esquerda, foi encontrado carcinoma complexo, apresentando células epiteliais malignas e intensa proliferação mioepitelial. A seta indica reação desmoplásica (o desenvolvimento de tecido conjuntivo está ligado às neoplasias malignas, associado a uma abundante matriz extracelular). Carcinoma complexo ou adenomioepitelioma maligno contém desenvolvimento de células mioepiteliais, células epiteliais, sendo difícil a diferenciação do carcinoma complexo do adenoma complexo ${ }^{15}$. No entanto, caso haja a presença de necrose e alta atividade mitótica, poderá facilitar o diagnóstico para carcinoma complexo.

Na lâmina (A), a glândula mamária abdominal cranial direita possui o carcinoma tubulopapilar: com proliferação de células epiteliais dispostas em padrão tubular. A seta indica a separação de tecido mamário e tecido neoplásico. Carcinoma tubulopapilar subdivide-se em carcinoma papilífero e carcinoma tubular. Os carcinomas papilíferos histologicamente caracterizam por proliferação epitelial papilar arborescente com região central de estroma fibrovascular, já as lesões papilares são classificadas como papilomas e carcinomas papilares ${ }^{15}$.

Na lâmina (D), glândula mamária torácica caudal esquerda e abdominal esquerda, foi visualizado o angiossarcoma que apresenta desenvolvimento de células endoteliais que formam vasos sanguíneos. Apresenta canais vasculares, que se anastomosam e infiltram as estruturas das glândulas.

Na lâmina (A), da glândula mamária abdominal caudal direita, há condições fibroblásticas (reação proliferativa). Na lâmina (B), da glândula mamária inguinal direita, foi encontrado um linfonodo intramamário (achado benigno). Na lâmina (C), a glândula mamária inguinal esquerda possuiu o adenoma complexo, com proliferação de células epiteliais e mioepiteliais. Na lâmina (D), a abdominal cranial direita apresentou um Lipoma mamário que contém estruturas ductais, com componentes vasculares e cartilagem madura. Neoplasia benigna originada dos lipócitos subcutâneos ${ }^{20}$.

Com relação aos tipos histológicos dos malignos encontrados, os mais comuns foram os carcinomas $91,6 \%$ (Gráfico 2). Tais resultados estão em consonância com estudos anteriores confirmando a prevalência bem mais alta para o carcinoma ${ }^{21}$, 22. Durante o período de setembro a outubro, foram coletadas amostras de neoplasias mamárias de 12 fêmeas após extirpação cirúrgica. No presente trabalho, a análise microscópica verificou, no que se refere ao comportamento histológico, uma frequência de $58,33 \%$ de neoplasias malignas. As neoplasias benignas evidenciaram um valor de $33,3 \%$. Neste trabalho, foram obtidos resultados semelhantes aos dados de outros pesquisadores ${ }^{12}$, que demonstraram uma frequência de $74,3 \%$ de tumores mamários malignos e um total de $16 \%$ dos casos benignos.

Gráfico 2. Frequência histológica absoluta de neoplasias malignas mais comuns. Viçosa (MG), 2015.

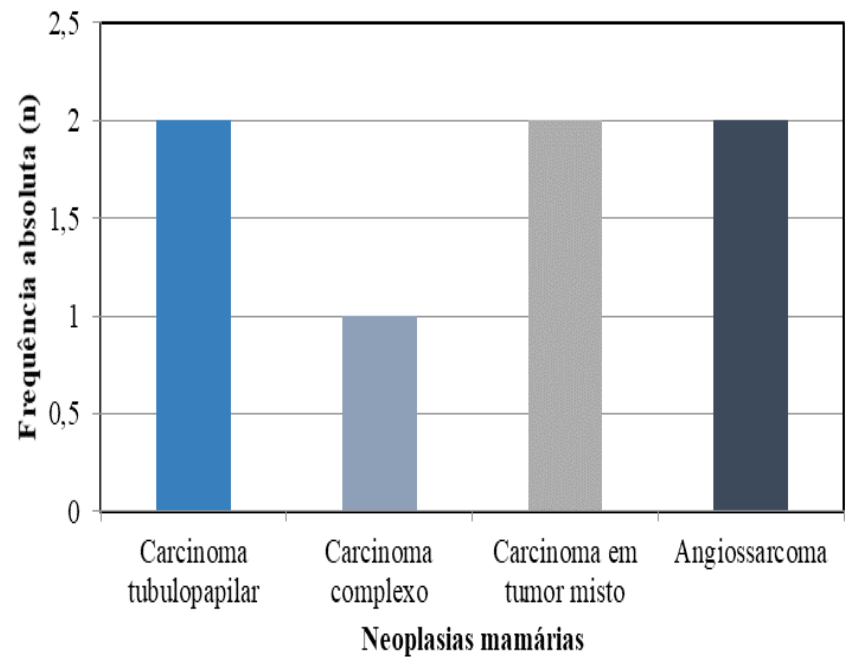

\section{CONCLUSÃO}

Por meio deste estudo, pode-se inferir que os tumores maiores são mais comuns e geralmente apresentam malignidade. Grande parte dos tumores da glândula mamária canina foi de comportamento maligno. Nota-se a importância de uma investigação criteriosa na análise das neoplasias mamárias na espécie canina, a fim de eleger uma terapia adequada e garantir um maior tempo de vida aos animais acometidos. O carcinoma, de acordo com os resultados obtidos, foi o tipo histológico mais comum. 


\section{REFERÊNCIAS}

1. Feliciano MAR, Silva AS, Peixoto RVR, Galera PD, Vicente WRR. Estudo clínico, histopatológico e imunoistoquímico de neoplasias mamárias em cadelas. Arq Bras Med Vet Zootec. 2012 Oct; 64(5):1094-100. doi: http://dx.doi.org/10.1590/ S0102-09352012000500002.

2. Jasckstet V. Marcadores prognósticos em tumores mamários de cadelas - revisão de literatura [monografia]. Brasília: UnB; 2013 [acesso 2017 jan 1]. Disponível em: http://bdm.unb.br/bitstream/10483/4777/1/2013 VanessaJasckstet.pdf.

3. Misdorp W. Tumors of the mammary gland. In: Meuten DJ. Tumors in domestic animals. 4th ed. lowa State Press; 2002. p. 575-606.

4. Guim TN. Determinação de fatores prognósticos para tumores mamários caninos [tese]. Pelotas (RS): Universidade Federal de Pelotas; 2011 [acesso 2017 jan 1]. Disponível em: http://repositorio.ufpel.edu.br/ bitstream/123456789/2562/1/tese_thomas_normanton_guim.pdf.

5. Silver IA. The anatomy of the mammary gland of the dog and cat. J Small Anim Pract. 1966 Nov; 7(11):689-96. PubMed PMID: 6009574.

6. Rosolem MC, Moroz LR, Rodigheri SM, Corrêa Neto UJ, Porto CD, Hanel JS. Análise comparativa entre citopatologia e histopatologia de casos atendidos em hospital veterinário escola no período de março de 2006 a março de 2011 . Rev Campo Digital [Internet]. Dez. 2012 [acesso 2017 jan 1]; 7(1):53-8. Disponíve| em: http://revista.grupointegrado.br/revista/index.php/campodigital/article/ view/1161/497

7. Cintra PP, Paula CAT, Calazans SG, Souza JL, Magalhães GM. Reclassificação e determinação do tipo histológico predominante em neoplasias mamárias caninas do hospital veterinário da Universidade de Franca nos anos de 2010 a 2012. Enciclop Biosf [Internet]. 2014 Jul [acesso 2017 jan 1]; 10(18): 260615. Disponível em: http://www.conhecer.org.br/enciclop/2014a/AGRARIAS/ reclassificacao.pdf.

8. Gomide PRS. Neoplasia mamária em cadelas: aspectos clínico-cirúrgicos [TCC]. Botucatu (SP): Universidade Estadual Paulista Júlio de Mesquita Filho; 2011 [acesso 2017 jan 1]. Disponível em: https://repositorio. unesp.br/bitstream/handle/11449/119299/gomide_prs_tcc_botfmvz. pdf?sequence $=1$ \&isAllowed $=\mathbf{y}$.

9. Prophet EB, Mills B, Arrington JB, Sobin LH. Laboratory methods in histotechnology. Washington, DC: Armed Forces Institute of Pathology; 1992. 279 p.

10. Brasil. Conselho Nacional de Controle de Experimentação Animal. Lei no 11.794 , de 8 de outubro de 2008. Regulamenta a criação e a utilização de animais em atividades de ensino e pesquisa científica. Diário Oficial [da] República Federativa do Brasil. 2008 Out. 09; Seção 1, p. 1.

11. Brasil. Conselho Nacional de Controle de Experimentação Animal. Decreto no 6.899, de 15 de julho de 2009. Dispõe sobre a composição do CONCEA, estabelece as normas para o seu funcionamento e de sua Secretaria-Executiva, cria o Cadastro das Instituições de Uso Científico de Animais - CIUCA, mediante a regulamentação da Lei no 11.794 , de 8 de outubro de 2008, que dispõe sobre procedimentos para o uso científico de animais, e dá outras providências. Diário Oficial [da] República Federativa do Brasil. 2009 Jul 16; Seção 1, p.2.

12. Zuccari DAPC, Santana AE, Rocha NS. Correlação entre a citologia aspirativa por agulha fina e a histologia no diagnóstico de tumores mamários de cadelas. Braz J Vet Res Anim Sci. 2001; 38(1):38-41. doi: http://dx.doi.org/10.1590/ S1413-95962001000100007.

13. Slatter D. Prolapso vaginal: manual de cirurgia de pequenos animais. Barueri: Manole; 1998.

14. Lana SE, Rutteman GR, Withrow SJ. Tumors of the mammary gland. In: Withrow SJ, Vail DM, editors. Small animal clinical oncology. 4th ed. St. Louis: Saunders Elsevier; 2007.

15. Cassali GD, Lavalle GE, Ferreira E, Estrela-Lima A, Nardi AB, Ghever C, et al. Consensus of the diagnosis, prognosis and treatment of canine mammary tumors - 2013. Braz J Vet Pathol [Internet]. 2014 July [acesso 2017 jan 1]; 7(2):38-69. Disponível em: http://bjvp.org.br/wp-content/uploads/2015/07/ DOWLOAD-FULL-ARTICLE-1-20881_2014_7_31_6_23.pdf.

16. Silva $A E$, Serakides $R$, Cassali GD. Carcinogênese hormonal e neoplasias hormônio-dependentes. Ciênc Rural [Internet]. 2004 [acesso 2017 jan 1]; 34(2): 625-33. Disponível em: http://www.scielo.br/pdf/cr/v34n2/a48v34n2.pdf.

17. Carvalho TB. Neoplasia mamária em cadelas: caracterização histopatológica e expressão de proteínas de estresse (HSP 72) [dissertação]. Viçosa (MG): Universidade Federal de Viçosa; 2006 [acesso 2017 jan 1]. Disponível em: http://locus.ufv.br/bitstream/handle/123456789/5119/texto\%20completo. pdf?sequence $=1$ \&isAllowed=y.

18. Oliveira JC Filho, Kommers GD, Masuda EK, Marques BMFPP, Fighera RA, Irigoyen LF, et al. Estudo retrospectivo de 1.647 tumores mamários em cães. Pesq Vet Bras. 2010; 30(2):177-85. doi: http://dx.doi.org/10.1590/S0100736X2010000200014

19. Chang SC, Chang CC, Chang TJ, Wong ML. Prognostic factors associated with survival two years after surgery in dogs with malignant mammary tumors: 79 cases (1998-2002). J Am Vet Med Assoc. 2005 Nov; 227(10):1625-9. PubMed PMID: 16313041.

20. Couto CG. Neoplasias selecionadas em cães e gatos. In: Nelson RW, Couto CG. Medicina interna de pequenos animais. 2. ed. Rio de Janeiro: Guanabara Koogan; 2001.

21. Karayannopoulou M, Kaldrymidou E, Constantinidis TC, Dessiris A. Histological grading and prognosis in dogs with mammary carcinomas: application of a human grading method. J Comp Pathol. 2005 Nov; 133(4): 24652. doi: https://doi.org/10.1016/j.jcpa.2005.05.003.

22. Goldschmidt M, Peña L, Rasotto R, Zappulli V. Classification and grading of canine mammary tumors. Vet Pathol. 2011 Jan; 48(1): 117-31. doi: https://doi. org/10.1177/0300985810393258.

\section{Como citar este artigo/How to cite this article:}

Alves ROD, Silva VF, Rodrigues LA, Xavier DCR, Silva PLN, Murta DVF. Caracterização Clínica e Histopatológica de Tumores Mamários em Cadelas Atendidas em Viçosa, MG. J Health Biol Sci. 2018 Jan-Mar; 6(1):17-22. 